\title{
Dimension of Fracture Surfaces
}

T. Ficker

The question of the universality of a dimension of fracture surfaces is discussed, and it is shown that such a general parameter may exist at least for a particular class of materials.

Keywords: fractal dimension, fracture surfaces, porous materials, compressive strength.

\section{Introduction}

Fracture surfaces are valuable sources of information on the structural composition and physical properties of materials. For these reasons they are a subject of interest for many research laboratories. Since the publication of the basic work by Mandelbrot and his co-workers [1] many authors have tried to correlate the fractal dimensions of fracture surfaces with the mechanical properties of materials. This effort has been impacted by the great complexity of these surfaces, especially in the case of composite porous materials. Cementitious materials have complex fracture surfaces that have been extensively studied [2-4].

The values of the fractal dimensions of a range of materials show only a narrow scatter, ranging from $\sim 2.0$ to $\sim 2.2$. Repeatedly determined dimensions of different fractured samples of the same materials have often resulted in identical values and this has led some authors [5] to the idea of a universal co-dimension (Hurst exponent) $H=0.8$ that characterizes fracture surfaces as a whole. Though this idea may invoke certain doubts at first sight [6], it should be carefully considered before it is rejected or accepted.

The aim of this paper is to investigate the concept of a universal co-dimension of fracture surfaces. As will be shown, this concept may be verified experimentally using fracture surfaces of porous materials in connection with their compressive strength. For this purpose, it is necessary first to derive corresponding relations for fractal porosity and fractal strength, and then to apply them to a particular material, in our case cement gel.

\section{Fractal porosity}

The large class of porous materials possesses at least one common feature, namely, they are composed of grains (particles, globules, etc.) of microscopic size $l$. The grains are usually arranged fractally with number distribution $N(l)$

$$
N(l)=\left(\frac{L}{l}\right)^{D}, \quad l<L .
$$

The porosity $P$ of the cluster

$$
P=1-\left(\frac{l}{L}\right)^{3-D}
$$

modifies its form if the porous material consists of more than one $(n+1)$ fractal cluster

$$
P=1-\sum_{i=0}^{n}\left(\frac{l_{i}}{L_{i}}\right)^{3-D_{i}} .
$$

However, relation (3) does not take into account the case of a composite material in which the fractal clusters of characteristic sizes $L_{i}$ can be stochastically scattered and mixed with other phases so that the size $\Lambda$ of the investigated sample may considerably exceed the cluster sizes $L_{i} \ll \Lambda$. In order to generalize relation (3), let use suppose that there are $m_{i}$ fractal clusters with dimension $D_{i}$ in the sample. Their volume fractions $\xi i=m_{i} L^{3} / \Lambda^{3}$ enable us to calculate the porosity of the whole sample, as follows

$$
P=1-\sum_{i=0}^{n} \xi_{i}\left(\frac{l_{i}}{L_{i}}\right)^{3-D_{i}} .
$$

Eq. (4) includes all possibilities of fractal, non-fractal $(D=3)$ or mixed arrangements of the solid environment surrounding the pores. Provided there are $n+1$ components distributed over the whole sample $\left(L_{i}=\Lambda, m_{i}=1, \xi_{i}=1\right)$, Eq. (4) then converts back to (3).

\section{Fractal compressive strength}

One of the most widely used relations for compressive strength $\sigma$ of a porous material is that of Balshin [7], though other relations [8] have also been proposed for this purpose. Balshin considered an ideal case when pores are not filled with an incompressible liquid and compressive strength $\sigma$ is directly dependent on the compactness $(1-P)$ of the material $\sigma=\sigma_{0}^{*}(1-P)^{k}$. However, as soon as the virtual incompressibility of the pore liquid is included together with some other factors, certain remaining strength $s_{0}$ appears as a constant when the porosity reaches a critical value $P_{\mathrm{cr}}$, i.e. $\sigma\left(P_{\mathrm{cr}}\right)=s_{0}$. The generalized Balshin function then reads

$$
\begin{aligned}
& \sigma=\sigma_{0}^{*}\left(1-\frac{P}{P_{\mathrm{cr}}}\right)^{k}+s_{0}=\sigma_{0}(1-P-b)^{k}+s_{0}, \\
& 0 \leq b=1-P_{\mathrm{cr}} \leq 1, \quad 0 \leq \sigma_{0}^{*} \leq \sigma_{0}=\frac{\sigma_{0}^{*}}{P_{\mathrm{cr}}^{k}},
\end{aligned}
$$

where $P$ is total porosity and $\sigma$ is compressive strength of the sample with a virtually incompressible fluid filling at least a part of its pore space.

Combining (4) and (5), the compressive strength of porous matter appears as a function of the fractal structure

$$
\sigma=\sigma_{0}\left[\sum_{i=0}^{n} \xi_{i}\left(\frac{l_{i}}{L_{i}}\right)^{3-D_{i}}-b\right]^{k}+s_{0} .
$$




\section{Dimensions of a fracture surface}

Generally, in the case of a mixed structure containing both fractal and non-fractal regions some of the dimensions $D_{i}$ are associated with volume (mass) fractals $\left(0<D_{i}<3\right)$ and some with non-fractal solid phases $\left(D_{i}=3\right)$. Similarly, when analyzing the fracture surfaces of such multicomponent materials, projections $\left(D_{i}^{*}\right)$ of the volume components $\left(D_{i}\right)$ appear on these surfaces. These projected surface "patterns" preserve the fractal or non-fractal characters of their volume parent objects, but their dimensions $D_{i}^{*}$ are smaller than those of the original objects. Provided a fracture surface has its own dimension $S$ and its morphology is "typical" rather than "special", the relation between $D_{i}^{*}$ and $D_{i}$ can be expressed [9] as follows

$$
D_{i}^{*}=\max \left\{0, D_{i}-(3-S\}, \quad D_{i}^{*} \leq S<3,\right.
$$

where $3-S$ is the codimension of the fracture surface. If the fracture surface were a perfect Euclidean plane $(S=2)$, expression (7) would lead to the well-known equation $D_{i}^{*}=D_{i}-1$. Using (7), the exponent $3-D_{i}$ in Eq. (6) can be replaced by $S-D_{i}^{*}$ and the generalized Balshin strength function now reads

$$
\sigma=\sigma_{0}\left[\sum_{i=0}^{n} \xi_{i}\left(\frac{l_{i}}{L_{i}}\right)^{S-D_{i}^{*}}-b\right]^{k}+s_{0} .
$$

This function may contain many parameters, so that it is very difficult to fit it to the experimental data, because there may be more than one "reliable" set of parameters $\sigma_{0},\left\{\xi_{i}\right\}_{i=1}^{n}$, $l_{i}, L_{i}, S, D_{i}^{*}, b, k, s_{0}$. Fortunately, the structure of a porous material often contains only one type of grain or - at least one grain type of fractal arrangement $(i=1)$ dominates over the solid remainder $(i=0)$, which is usually of a non-fractal character $\left(D_{0}=3 \Rightarrow D_{0}^{*}=S\right)$

$$
\begin{aligned}
& \sigma=\sigma_{0}\left[\xi_{1}\left(\frac{l_{1}}{L_{1}}\right)^{S-D_{1}^{*}}+\left(\xi_{0}-b\right)\right]^{k}+s_{0}= \\
& \sigma_{0}\left[\xi_{1} \exp \left(\frac{S-D_{1}^{*}}{A}\right)-\gamma\right]^{k}+s_{0}, \\
& A=\frac{1}{\ln \left(\frac{L_{1}}{l_{1}}\right)}, \quad \gamma=\left(b-\xi_{0}\right) .
\end{aligned}
$$

To our knowledge, the fractal analyses of fracture surfaces published so far have not distinguished between the dimensions $D_{i}^{*}$ of the projected objects and the dimension $S$ of the fracture surface itself. Here, for the first time clear differences between these two kinds of dimensions are specified and discussed. Although the fracture surface dimension $S$ as a separate parameter and independent of the inner fractal structure may seem to be rather vague, it nevertheless has a clear interpretation as the fracture surface dimension that would be directly measurable if the sample were fully compact (non-porous, i.e. $b=0, s_{0}=0$ ), and, therefore, non-fractal $\left(D_{i}=3 \Rightarrow D_{i}^{*}=S\right)$, which means a perfect Euclidean body. Nevertheless, the surface itself may be a fractal with non-integer $S$, which should even be expected in most cases.

The foregoing equations and discussion have shown that fracture surfaces bear information about the multi-component volume structure that is projected on the surface as the spectrum of surface patterns $\left\{D_{i}^{*}\right\}_{i=0}^{n}$ that preserve their fractal or non-fractal origins. The fracture surface has its own dimension independent of from the dimensions of the projected surface patterns. These facts require a strict distinction between dimensions $D_{i}^{*}$ and $S$, and also among $D_{i}^{*}$ themselves. This can be done either locally (e.g. using a microscopic technique) or globally within restricted fractal scales $\left\langle l_{i}, L_{i}\right\rangle$ if, of course, there are no overlaps between the scales.

\section{Experimental results}

To illustrate the soundness of the concept of fractal compressive strength (9) and the concept of, their application to hydrated Portland cement is presented in the following paragraphs. Seventy-two samples of hydrated ordinary Portland cement paste of various water-to-cement ratios $r(0.4,0.6,0.8$, $1.0,1.2,1.4)$ were prepared. After 28 days of hydration the samples were subjected to three-point bending and were fractured. The fracture surfaces were used for further fractal analysis. The 3-D digital reconstruction of the fracture surfaces (Fig. 1) was performed using a confocal microscope and then a series of horizontal sections (contours) were analyzed with resolution $0.04 \mu \mathrm{m}^{2} /$ pixel by means of the standard box-counting method [10-12] to obtain a representative $D^{*}$ for the particular self-affine surface. The box-counting analyses were performed in the length interval $\langle 0.2 \mu \mathrm{m}, 30 \mu \mathrm{m}\rangle$. The

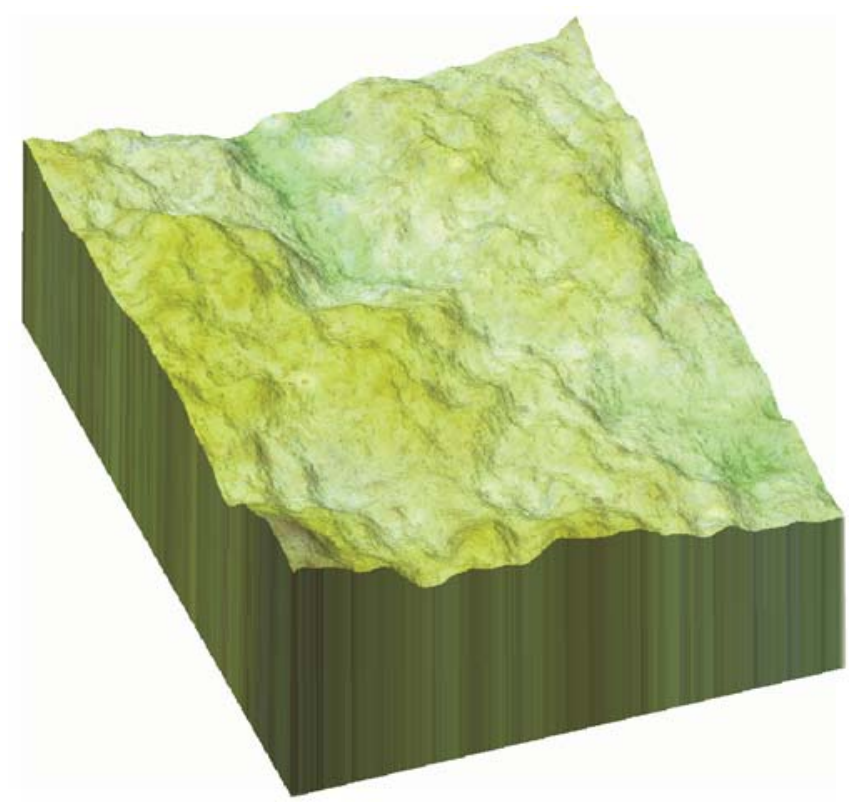

Fig. 1: Digital 3-D reconstruction of fracture surface (confocal microscopy: $0.34 \mathrm{~mm} \times 0.21 \mathrm{~mm}$; total magnification $600 \times$ ) 


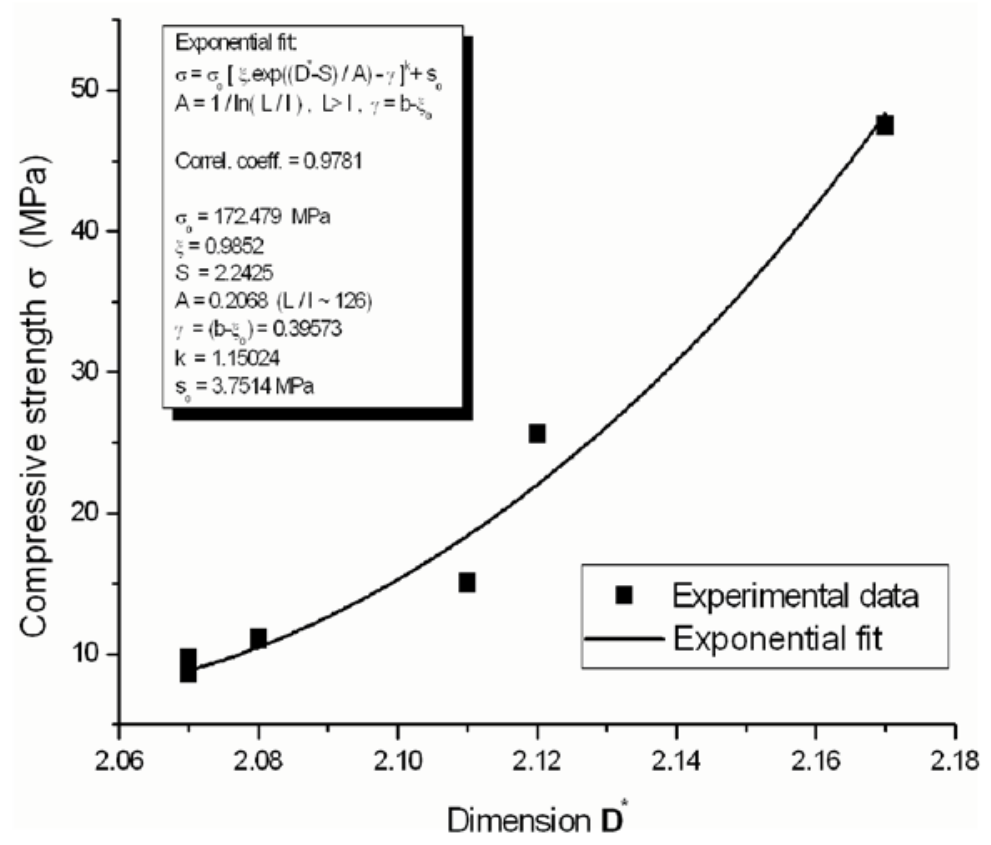

Fig. 2: Dependence of compressive strength on fractal dimension with cement paste

second parts of the fractured samples were cut into small cubes and subjected to destructive tests to determine their compressive strength values.

Samples prepared with different water-to-cement ratios $r$ possess different porosity. From cement technology it is well-known that with increasing ratio $r$ the porosity increases exponentially. Naturally, this will change the dimensions of the projected patterns $D^{*}$. Six groups of samples with different $r$ means six different $D^{*}$ at which we are able to measure the dependence $\sigma\left(D^{*}\right)$ and check it according to Eq. (9). The result is shown in Fig. 2, along with all the fitting parameters. Since the assumed analytical form (9) of dependence $\sigma\left(D^{*}\right)$ has been reproduced well, we may conclude that compressive strength is one of those mechanical quantities whose value is "coded" in the surface arrangement of the fractured samples of porous materials.

Dimension $S$ of the fracture surfaces of hydrated cement paste proved to be near to 2.24 , indicating a fractally corrugated surface onto which another fractal component $D^{*} \in\langle 2.075 ; 2.170\rangle$ is projected $(\xi \approx 1)$. The range of values of $D^{*}$ is in full accord with other authors [2-6]. The contribution of the non-fractal solid phase $\left(\xi_{0} \rightarrow 0\right)$ is negligible $\left(\xi \gg \xi_{0}\right)$. As far as dimension $D$ of the original volume (mass) fractal of the cement paste used is concerned, its value is rather high $D \in\langle 2.835 ; 2.930\rangle$ but this was expected due to the low dimension $D^{*}$ of the corresponding projection and the high value of the co-dimension, i.e. Hurst exponent $H$, of the fracture surface itself $H=3-S \approx 0.76$. The value of $D$ near to 2.90 agrees with the dimension of the cement gel within the hydrated cement paste having $r=0.4$ and exposed to $\sim 55 \% \mathrm{RH}$ [13], [14].

\section{Conclusion}

It has been shown that the fracture surface possesses its own dimension $S$ which is independent on the dimensions of the patterns projected on this surface. Dimension $S$ seems to depend more on the fracture process itself than on structural components. This feature may partly support the claims of several authors [5] that there is a universal Hurst exponent $H=0.8$ for a wide class of materials. Now it is clear that if such a universal exponent really exists, it is not as a co-dimension of the surface projected patterns $3-D^{*}$ but as a co-dimension of the fracture surface itself $H=3-S$. In our case $H \approx 0.76$, which is almost identical with the previously anticipated [5] value 0.8 .

Dimension $S$ can be determined not only as the difference $3-\left(D-D^{*}\right)$ but also as a "byproduct" when measuring a convenient physical quantity, in our case compressive strength. Dimension $S$ of fracture surfaces may be interpreted as the dimension that would be directly measurable if the sample were fully compact (non-porous and non-fractal), i.e. a perfect Euclidean body. Nevertheless, the fracture surface itself may be a fractal with non-integer $S$, which should be expected most cases.

\section{Acknowledgment}

This work was supported by an Internal Grant assigned by the Faculty of Civil Engineering, Brno University of Technology, in 2007.

\section{References}

[1] Mandelbrot, B. B., Passoja, D. E., Panllay, A. J.: Fractal Character of Fracture Surfaces of Metals, Nature (London) Vol. 308 (1984), p. 721-722.

[2] Yan, A., Wu, K.-R., Zhang, D., Yao, W.: Effect of Fracture Path on the Fracture Energy of High-Strength Concrete, Cem. Concr. Res. Vol. 25 (2003), p. 153-157.

[3] Wang, Y., Diamond, S.: A Fractal Study of the Fracture Surfaces of Cement Pastes and Mortars Using a Stereo- 
scopic SEM Method, Cem. Concr. Res. Vol. 31 (2001), p. 1385-1392.

[4] Prokopski, G., Langier, B.: Effect of Water/Cement Ratio and Silica Fume Addition on the Fracture Toughness and Morphology of Fractured Surfaces of Gravel Concretes, Cem. Concr. Res. Vol. 30 (2000), p. 1427-1433.

[5] Bouchaud, E., Lapasset, G., Plan s, J.: Fractal Dimension of Fractured Surfaces: a universal value?, Europhys. Lett. Vol. 13 (1990), p. 73-79.

[6] Carpinteri, A., Chiaiai, B., Invernizzi, S.: Comments on "Direct fractal measurement of fracture surfaces", Int. Sol. Struct. Vol. 37 (2000), p. 4623-4625.

[7] Balshin, M. Y.: Zavisimost mechaniceskich svojstv poroskovych metalov ot poristnosti i predelnyje svojstva poristych metalokeramiceskich materialov, Dokl. Akad. Nauk SSSR (in Russian), Vol. 67 (1949), p. 831-834.

[8] Narayanan, N., Ramamurthy, K.: Structure and Properties of Aerated Concrete: a Review, Cem. Concr. Res. Vol. 22 (2000), p. 321-329.

[9] Falconer, K. J., Professor of Mathematics, University of St. Andrews, Scotland - private communication.

[10] Ficker, T.: Electrostatic Discharges and Multifractal Analysis of their Lichtenberg Figures, J. Phys. D: Appl. Phys. Vol. 32 (1999), p. 219-226.
[11] Ficker, T.: Fractal Morphology of Partial Microdischarge Spots on Dielectric Barriers, IEEE Trans. Diel. El. Insul. Vol. 10 (2003), p. 700-707.

[12] Ficker, T.: Electrostatic Microdischarges on the Surface of Electrets, J. Phys. D: Appl. Phys. Vol. 38 (2005), p. 483-489.

[13] Winslow, D., Bukowski, J. M., Young, J. F.: The Fractal Arrangement of Hydrated Cement Paste, Cem. Concr. Res. Vol. 25 (1995), p. 147-156.

[14] Boddoe, R. E., Lang, K.: Effect of Moisture on Fractal Dimension and Specific Surface of Hardened Cement Paste by Small-Angle X-ray Scattering, Cem. Concr. Res. Vol. 24 (1994), p. 605-612.

Prof. RNDr. Tomáš Ficker, DrSc. phone: +420541 147661

e-mail: ficker.t@fce.vutbr.cz

Department of Physics

University of Technology

Faculty of Civil Engineering

Žižkova 17

66237 Brno, Czech Republic 